\title{
Effects of Escitalopram on Plasma Concentrations of Aripiprazole and its Active Metabolite, Dehydroaripiprazole, in Japanese Patients
}

\author{
Authors \\ Affiliations \\ Key words \\ - aripiprazole \\ - dehydroaripiprazole \\ - escitalopram \\ - paroxetine \\ drug interaction
}

\author{
K. Nemoto ${ }^{1}$, K. Mihara ${ }^{1}$, A. Nakamura ${ }^{1}$, G. Nagai ${ }^{1}$, S. Kagawa ${ }^{1}$, T. Suzuki ${ }^{1}{ }^{1}$, T. Kondo
}

Department of Neuropsychiatry Faculty of Medicine, University of the Ryukyus, Nishihara, Okinawa, Japan Department of Pharmacy Faculty of Medicine, University of the Ryukyus, Nishihara, Okinawa, Japan $\begin{array}{ll}\text { received } & 06.02 .2014 \\ \text { revised } & 20.03 .2014 \\ \text { accepted } & 20.03 .2014\end{array}$

Bibliography

DOI http://dx.doi.org/

10.1055/s-0034-1372644

Published online ahead of print:

24 April 2014

Pharmacopsychiatry 2014;

47: 101-104

(c) Georg Thieme Verlag KG

Stuttgart · New York

ISSN 0176-3679

\section{Correspondence}

\section{K. Mihara, MD, PhD}

Department of Neuropsychiatry Faculty of Medicine

University of the Ryukyus

207 Uehara

Nishihara-cho

Okinawa 903-0215

Japan

Tel.: + 81/98/895 1157

Fax: $+81 / 98 / 8951419$

miharak@med.u-ryukyu.ac.jp

\section{Abstract \\ $\nabla$}

Introduction: The effects of escitalopram $(10 \mathrm{mg} / \mathrm{d})$ coadministration on plasma concentrations of aripiprazole and its active metabolite, dehydroaripiprazole, were studied in 13 Japanese psychiatric patients and compared with those of paroxetine $(10 \mathrm{mg} / \mathrm{d})$ coadministration. Methods: The patients had received 6-24 mg/d of aripiprazole for at least 2 weeks. Patients were randomly allocated to one of 2 treatment sequences: paroxetine-escitalopram ( $n=6)$ or escitalopram-paroxetine $(n=7)$. Each sequence consisted of two 2-week phases. Plasma concentrations of aripiprazole and dehydroaripiprazole were measured using liquid chromatography with mass spectrometric detection.

\section{Introduction \\ $\nabla$}

Aripiprazole is a novel second-generation antipsychotic drug with a pharmacological profile of dopamine and $5-\mathrm{HT}_{1 \mathrm{~A}}$ partial agonisms, and $5-\mathrm{HT}_{2 \mathrm{~A}}$ antagonism [1]. The efficacy of aripiprazole has been established for the treatment of schizophrenia [2]. It is also reported that aripiprazole is efficacious for a range of symptoms associated with pervasive developmental disorders [3]. Aripiprazole undergoes N-dealkylation, hydroxylation and dehydrogenation, yielding its active metabolite [4], dehydroaripiprazole. Dehydroaripiprazole is further hydroxylated and N-dealkylated [4]. Kubo et al. [5] have suggested that the cytochrome (CYP) 2D6 enzyme is involved in the metabolism of aripiprazole after a single oral dose of aripiprazole. This finding has been confirmed by the authors' previous study [6] on the steady-state plasma concentration of the drug in subjects with different CYP2D6 genotypes. Because dehydroaripiprazole has similar pharmacological properties as the parent com-
Results: Plasma concentrations of aripiprazole and the sum of aripiprazole and dehydroaripiprazole during paroxetine coadministration were 1.7-fold (95\% confidence intervals [CI], 1.3-2.1, $\mathrm{p}<0.001$ ) and 1.5-fold (95\% CI 1.2-1.9, $\mathrm{p}<0.01$ ) higher than those values before the coadministration. These values were not influenced by escitalopram coadministration (1.3-fold, 95\% CI 1.1-1.5 and 1.3-fold, 95\% CI 1.0-1.5). Plasma dehydroaripiprazole concentrations remained constant during the study.

Conclusion: The present study suggests that low doses of escitalopram can be safely coadministered with aripiprazole, at least from a pharmacokinetic point of view.

pound [2], and the plasma concentration of the metabolite amounts to $40 \%$ of that of aripiprazole at steady state [7], the sum of the 2 compounds is considered to contribute to the overall antipsychotic efficacy.

Selective serotonin reuptake inhibitors (SSRI) are widely used for treatment of several psychiatric disorders. SSRI augmentation of antipsychotics is considered to be one of the options for ameliorating negative symptoms of schizophrenia [8]. In clinical settings, SSRI can be coadministered to patients with schizophrenia or pervasive developmental disorders being treated with antipsychotic drugs for the alleviation of depressive and/ or obsessive-compulsive symptoms. The authors have already studied the effect of paroxetine, which is a potent inhibitor of CYP2D6, on plasma concentrations of aripiprazole and dehydroaripiprazole in Japanese patients with schizophrenia [9], showing that even low doses of paroxetine coadministration increase plasma concentrations of aripiprazole and the sum of aripiprazole and dehydroaripiprazole. 
Escitalopram is a weak inhibitor of CYP2D6 [10]. Previous studies $[11,12]$ suggested that escitalopram increases serum concentrations of aripiprazole. However, these reports [11,12] were based on group comparisons between patients who were and were not taking escitalopram, utilizing therapeutic drug monitoring data. In addition, one of the reports [11] did not deal with serum dehydroaripiprazole concentrations. Therefore, the authors aimed to intraindividually examine the effects of escitalopram coadministration on plasma concentrations of aripiprazole and dehydroaripiprazole, in comparison with paroxetine coadministration.

\section{Materials and Methods}

$\nabla$

\section{Patients}

The study included 13 Japanese inpatients ( 8 males and 5 females) who all fulfilled the DSM-IV-TR criteria for schizophrenia $(n=9)$ or pervasive developmental disorders $(n=4)$. They were physically healthy without any history of substance abuse, neurological disorder, delirium or dementia and without any clinically significant abnormalities, including clinical laboratory examinations, electrocardiography and electroencephalography. The mean \pm SD age and body weight were $34.5 \pm 9.7$ years and $62.3 \pm 13.4 \mathrm{~kg}$, respectively. There were no smokers or heavy drinkers among the patients. The study was approved by the Ethics Committee of the University of the Ryukyus, and all the patients had given written informed consent to participate in this study.

\section{Study design}

All the subjects had received aripiprazole for at least 2 weeks, because it has been shown that plasma concentrations of both aripiprazole and dehydroaripiprazole reach steady-state by 2 weeks of repeated oral administrations [13]. The daily dose was fixed at $24 \mathrm{mg}$ in 7 cases, $12 \mathrm{mg}$ in 3 cases, and $6 \mathrm{mg}$ in 3 cases during the study period and was given once a day at $12: 30 \mathrm{pm}$. Except for $2-4 \mathrm{mg} / \mathrm{d}$ flunitrazepam $(\mathrm{n}=4)$ and $4 \mathrm{mg} / \mathrm{d}$ biperiden $(n=2)$ the patients received no drugs. Female patients did not receive oral contraceptives. The doses of these coadministered drugs were also fixed throughout the study period. The subjects were randomly allocated to one of 2 treatment sequences, either escitalopram-paroxetine at $7 \mathrm{pm}(\mathrm{n}=6)$ or paroxetine-escitalopram at $7 \mathrm{pm}(\mathrm{n}=7)$. Each sequence consisted of two 2-week phases, with no washout period between the 2 phases. The daily dose of escitalopram or paroxetine was $10 \mathrm{mg}$. The patients' adherence was confirmed by the nursing staff. Blood samples were taken before the coadministration, 2 weeks after the first SSRI, and 2 weeks after the second SSRI. All the samples were taken at $8 \mathrm{am}$. On the same days, the severity of illness and extrapyramidal adverse effects were evaluated using the Clinical Global Impressions (CGI) [14] and Drug-Induced Extrapyramidal Symptoms Scale (DIEPSS) [15], respectively.

\section{Laboratory methods}

Plasma concentrations of aripiprazole and dehydroaripiprazole were measured using the liquid chromatography method with mass spectrometric detection described by Kubo et al. [16]. The lower limit of quantification for both compounds was $0.1 \mathrm{ng} / \mathrm{mL}$ using $0.4 \mathrm{~mL}$ of plasma, and the interassay coefficient of variation was less than $8 \%$ at $0.2 \mathrm{ng} / \mathrm{mL}$ for both aripiprazole and dehydroaripiprazole. $1 \mathrm{ng} / \mathrm{mL}$ of aripiprazole and dehydroaripi- prazole are corresponding to $2.23 \mathrm{nM}$ and $2.24 \mathrm{nM}$, respectively. The plasma drugs concentrations were normalized to a dose of $24 \mathrm{mg} / \mathrm{d}$, and the values were used in statistical analyses.

DNA was isolated from peripheral leukocytes using QIA-amp DNA Blood Maxi (QIAGEN, Tokyo, Japan). Long polymerase chain reaction analysis was used to detect the CYP2D $6 * 5\left({ }^{*} 5\right)$ allele [17]. The CYP2D6 $\left({ }^{*} 4\right), C Y P 2 D 6^{*} 10\left({ }^{*} 10\right)$ and CYP2D6*14(*14) alleles were identified by polymerase chain reaction analyses as described by Heim and Meyer [18], Johansson et al. [19] and Kubota et al. [20], respectively. Alleles other than *4, ${ }^{*} 5$, ${ }^{*} 10$ and ${ }^{*} 14$ were defined as wild type $(w t)$. The CYP2C19*2 and CYP2C19*3 alleles were detected as described by Kimura et al. [21]. These mutated alleles and the $w t$ allele, which are regarded as predominant over other CYP2D6 and CYP2C19 alleles, were selected based on the frequency of CYP2D6 [22] and CYP2C19 [21] alleles in a Japanese population.

\section{Statistics}

The Friedman test followed by the Wilcoxon signed-rank test with Bonferroni correction were used for the comparison of the plasma concentrations of aripiprazole and dehydroaripiprazole and the scores of clinical assessments. A 2-tailed $P$ value of less than 0.05 was regarded as statistically significant. SSPS 19.0 for Windows (SPSS, Japan Inc, Tokyo, Japan) was used for these statistical analyses.

\section{Results}

$\nabla$

- Table 1 shows the changes in the plasma concentrations of aripiprazole, dehydroaripiprazole, the sum of aripiprazole and dehydroaripiprazole, and the CGI and DIEPSS scores before and after the 2 treatment sequences. The mean plasma concentrations of aripiprazole and the sum of the 2 compounds during the coadministration of paroxetine were 1.7 -fold (95\% confidence intervals [CI], 1.3-2.1, p<0.001) and 1.5-fold (95\% CI 1.2-1.9, $\mathrm{p}<0.01$, $\odot$ Fig. 1) higher than those values before the coadministration. Those values were not influenced by escitalopram coadministration (1.3-fold, 95\% CI 1.1-1.5 and 1.3-fold, 95\% CI 1.0-1.5). The mean plasma concentrations of dehydroaripiprazole were constant throughout the study period.

The patients had the following genotypes for CYP2D6 and CYP2C19: *10/wt $(\mathrm{n}=11), w t / w t(\mathrm{n}=1)$, and ${ }^{*} 10 /{ }^{*} 10(\mathrm{n}=1) ;{ }^{*} 2 /{ }^{*} 2$ $(\mathrm{n}=5), w t / w t(\mathrm{n}=4),{ }^{*} 2 / w t(\mathrm{n}=3)$, and ${ }^{*} 3 / w t(\mathrm{n}=1)$ The genotypes were not associated with the increases of the plasma concentrations (data not shown). The mean CGI and DIEPPS scores remained unchanged during the study.

\section{Discussion}

$\nabla$

In the present study, escitalopram coadministration did not significantly change the mean plasma concentrations of aripiprazole. The finding suggests that escitalopram does not affect the metabolism of aripiprazole. There were 2 plausible explanations for this negative finding. The first is due to the less inhibitory effect of escitalopram on the CYP2D6 activity [10] compared with paroxetine [9]. The second is that aripiprazole may have a higher affinity for this isoenzyme than escitalopram. The present result is not consistent with previous reports $[11,12]$ based on group comparisons suggesting that escitalopram increased serum aripiprazole concentrations. However, this study may be 
Table 1 Plasma concentrations normalized to a dose of $24 \mathrm{mg} / \mathrm{d}$ of ARI, DARI, and the sum of ARI and DARI, and CGI and DIEPSS scores before and after paroxetine or escitalopram coadministration in Japanese psychiatric inpatients.

\begin{tabular}{|llll} 
& Before coadministration & Paroxetine coadministration & \multicolumn{1}{c}{ Escitalopram coadministration } \\
\hline ARI $(\mathrm{ng} / \mathrm{mL})$ & $514 \pm 156$ & $862 \pm 336^{*}$ & $661 \pm 238$ \\
\hline ratio to baseline & & $1.7(1.3-2.1)$ & $1.3(1.1-1.5)$ \\
\hline DARI $(\mathrm{ng} / \mathrm{mL})$ & $260 \pm 96$ & $273 \pm 117$ & $274 \pm 86$ \\
\hline ratio to baseline & & $1.2(0.7-1.8)$ & $1.2(0.9-1.5)$ \\
\hline ARI + DARI $(\mathrm{ng} / \mathrm{mL})$ & $774 \pm 231$ & $1135 \pm 443 \dagger$ & $934 \pm 312$ \\
ratio to baseline & & $1.5(1.2-1.9)$ & $1.3(1.0-1.5)$ \\
\hline CGI & $3.5 \pm 0.8$ & $3.1 \pm 1.2$ & $3.0 \pm 1.0$ \\
\hline DIEPSS & $2.8 \pm 2.0$ & $3.0 \pm 3.5$ & $2.7 \pm 2.9$
\end{tabular}

$\mathrm{ARI}=$ aripiprazole; $\mathrm{DARI}=$ dehydroaripiprazole; $\mathrm{CGI}=$ Clinical Global Impressions; DIEPSS=drug-induced extrapyramidal symptoms scale. The values of concentrations and scores are mean \pm SD. The ratios to baseline are mean ( $95 \%$ confident intervals)

${ }^{*} \mathrm{p}<0.001$ compared with before coadministration

$\uparrow \mathrm{p}<0.01$ compared with before coadministration

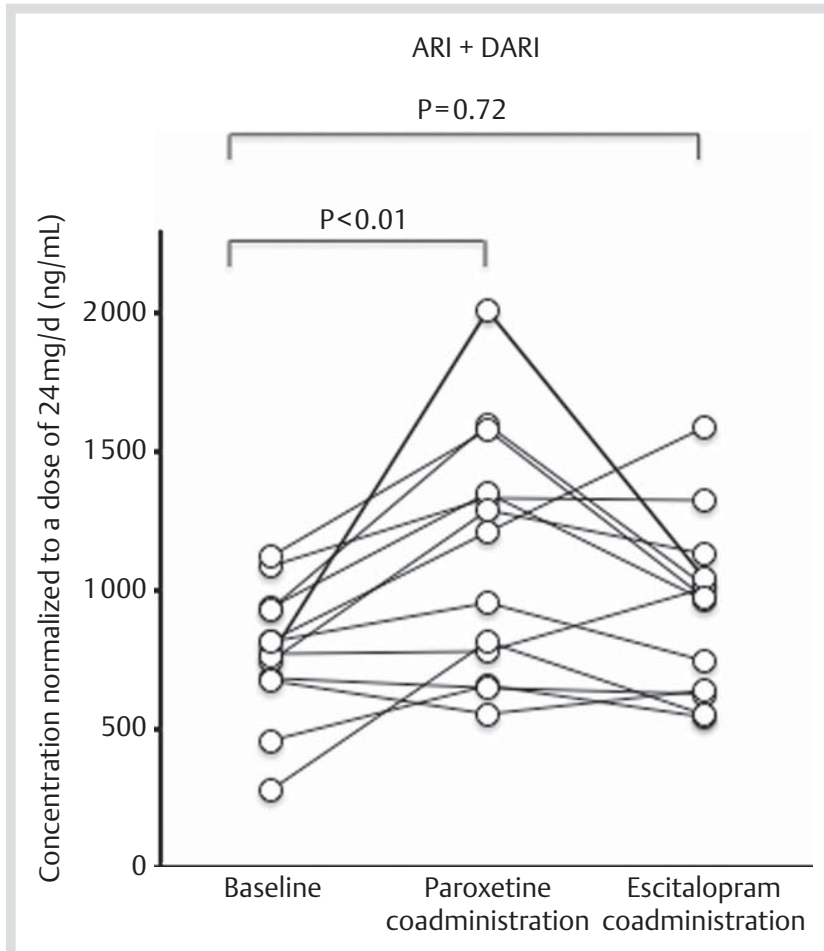

Fig. 1 Intraindividual changes in plasma concentrations normalized to a dose of $24 \mathrm{mg} / \mathrm{d}$ for the sum of aripiprazole and dehydroaripiprazole before and after paroxetine or escitalopram coadministration. ARI=aripiprazole; DARI= dehydroaripiprazole.

more reliable than the previous ones $[11,12]$ because the present study prospectively examined the effect of escitalopram on aripiprazole metabolism intraindividually. Although type II error cannot be entirely ruled out due to small number of subjects in this study, the impact of escitalopram coadministration on the metabolism of aripiprazole would still remain marginal at best. Thus, escitalopram can be safely coadministered to aripiprazole in Japanese patients, at least from a pharmacokinetic point of view.

A 2-week coadministration of paroxetine $10 \mathrm{mg} / \mathrm{d}$ significantly increased the mean plasma concentrations of aripiprazole. The result replicates the authors' previous study [9] showing that 1 -week coadministration of paroxetine $10 \mathrm{mg} / \mathrm{d}$ significantly increased those values. It is confirmed that even a low dose of paroxetine inhibits aripiprazole metabolism via CYP2D6 inhibition. However, the degree of increase in plasma aripiprazole concentrations is 1.7 -fold, which is similar to the 1.5 -fold found in the previous study [9]. Therefore, the degree of increase in aripiprazole concentration by paroxetine $10 \mathrm{mg} / \mathrm{d}$ may be limited to 2-fold at best.

The mean plasma concentrations of dehydroaripiprazole remained constant during the study period, which shows that neither paroxetine nor escitalopram affect the metabolism of dehydroaripiprazole. This is in line with the authors' previous studies which showed no involvement of * 10 in the steady-state plasma concentrations of dehydroaripiprazole [6], and that the addition of paroxetine does not influence the metabolism of dehydroaripiprazole [9]. Therefore, it is confirmed that CYP2D6 activity is not related to plasma concentration of dehydroaripiprazole.

Paroxetine significantly increased the sum of aripiprazole and dehydroaripiprazole, while escitalopram did not. However, no significant changes in CGI and DIEPSS scores were observed during the study. There are 4 possible reasons for the result, which are associated with limitations in this study. First, the subjects were heterogeneous in age, gender, and diagnoses. Second, the duration of SSRI coadministration might not have been long enough to detect pharmacodynamic changes. Third, the doses of aripiprazole were not unified. Fourth, the sample size was small. Thus, the present study should be replicated with a larger number of homozygous subjects whose clinical symptoms are more precisely evaluated after longer periods of SSRI coadministration.

In conclusion, escitalopram can be safely coadministered to aripiprazole in Japanese patients, at least from a pharmacokinetic point of view.

\section{Acknowledgements \\ $\nabla$}

Authentic aripiprazole and dehydroaripiprazole were kindly provided by Otsuka Pharmaceutical, Co, Ltd, Tokyo, Japan. We thank Mr. David Webb for his helpful advice.

\section{Conflict of Interest \\ $\nabla$}

Dr. Mihara has received honoraria from GlaxoSmithKline and Otsuka. Dr. Nakamura has received honoraria from Dainippon Sumitomo Pharma and Otsuka. Dr. Kondo has received honoraria from GlaxoSmithKline and Otsuka. 


\section{References}

1 Shapiro DA, Renock S, Arrington E et al. Aripiprazole, a novel atypical antipsychotic drug with a unique and robust pharmacology. Neuropsychopharmacology 2003; 28: 1400-1411

2 Harrison TS, Perry CM. Aripiprazole: a review of its use in schizophrenia and schizoaffective disorder. Drugs 2004; 64: 1715-1736

3 Jordan I, Robertson D, Catani $M$ et al. Aripiprazole in the treatment of challenging behaviour in adults with autism spectrum disorder. Psychopharmacolgy 2012; 223: 357-360

4 Caccia S. N-Dealkylation of arylpiperazine derivatives: disposition and metabolism of the 1-aryl-piperazines formed. Curr Drug Metab 2007; 8: 612-622

5 Kubo M, Koue T, Inaba A et al. Influence of itraconazole co-administration and CYP2D6 genotype on the pharmacokinetics of the new antipsychotic aripiprazole. Drug Metab Pharmacokinet 2005; 20: 55-64

6 Suzuki T, Mihara K, Nakamura A et al. Effects of the CYP2D6*10 allele on the steady-state plasma concentrations of aripiprazole and its active metabolite, dehydroaripiprazole, in Japanese patients with schizophrenia. Ther Drug Monit 2011; 33: 21-24

7 Kirschbaum KM, Müller MJ, Malevani J et al. Serum levels of aripiprazole and dehydroaripiprazole, clinical response and side effects. World J Biol Psychiatry 2008; 9: 212-218

8 Silver $H$. Selective serotonin reuptake inhibitor augmentation in the treatment of negative symptoms of schizophrenia. Int Clin Psychopharmacol 2003; 18: 305-313

9 Nemoto K, Mihara K, Nakamura A et al. Effects of paroxetine on plasma concentrations of aripiprazole and its active metabolite, dehydroaripiprazole, in Japanese patients with schizophrenia. Ther Drug Monit 2012; 34: 188-192

10 Preskorn SH, Greenblatt DJ, Flockhart D et al. Comparison of duloxetine, escitalopram, and sertraline effects on cytochrome P450 2D6 function in healthy volunteers. J Clin Psychoph/armacol 2007; 27: 28-34

11 Castberg I, Spigset 0 . Effects of comedication on the serum levels of aripiprazole: evidence from a routine therapeutic drug monitoring service. Pharmacopsychiatry 2007; 40: 107-110
12 Waade RB, Christensen H, Rudberg I et al. Influence of comedication on serum concentrations of aripiprazole and dehydroaripiprazole. Ther Drug Monit 2009; 31: 233-238

13 Kubo M, Koue T, Maune $H$ et al. Pharmacokinetics of aripiprazole, a new antipsychotic, following oral dosing in healthy adult Japanese volunteers: influence of CYP2D6 polymorphism. Drug Metab Pharmacokinet 2007; 22: 358-366

14 Guy W. Clinical Global Impressions. Guy W (ed.). ECDEU Assessment Manual for Psychopharmacology. Revised. Rockville: US Department of Health, Education and Welfare; 1976; 217-222

15 Inada T, Yagi G, Miura S. Extrapyramidal symptom profiles in Japanese patients with schizophrenia treated olanzapine or haloperidol. Schizophr Res 2002; 57: 227-238

16 Kubo M, Mizooku Y, Hirao Y et al. Development and validation of an LC-MS/MS method for the quantitative determination of aripiprazole and its main metabolite, OPC-14857, in human plasma. J Chromatography B 2005; 822: 294-299

17 Steen VM, Andreassen OA, Daly AK et al. Detection of the poor metabolizer-associated CYP2D6(D) gene deletion allele by long-PCR technology. Pharmacogenetics 1995; 5: 215-223

18 Heim M, Meyer U. Genotyping of poor metabolizers of debrisoquine by allele-specific PCR amplification. Lancet 1990; 336: 529-532

19 Johansson I, Oscarson M, Yue QY et al. Genetic analysis of the Chinese cytochrome P4502D locus: characterization of variant CYP2D6 genes present in subjects with diminished capacity for debrisoquine hydroxylation. Mol Pharmacol 1994; 46: 452-459

20 Kubota T, Yamaura Y, Ohkawa $N$ et al. Frequencies of CYP2D6 mutant alleles in a normal Japanese population and metabolic activity of dextromethorphan O-demethylation in different CYP2D6 genotypes. $\mathrm{Br} \mathrm{J}$ Clin Pharmacol 2000; 50: 31-34

21 Kimura M, Ieiri I, Urae K et al. Genetic polymorphism of cytochrome P450s, CYP2C19 and CYP2C9 in a Japanese population. Ther Drug Monit 1998; 20: 243-247

22 Sakuyama K, Sasaki T, Ujiie S et al. Functional characterization of 17 CYP2D6 allelic variants (CYP2D6.2, 10, 14A-B, 18, 27, 36, 39, 47-51, 53-55, and 57). Drug Metab Dispos 2008; 36: 2460-2467 